\title{
Dynamic performance simulation and control of an aeroengine by using NARX models
}

\author{
Maria Grazia De Giorgi ${ }^{1}$, Antonio Ficarella and Marco Quarta \\ ${ }^{1}$ Department of Engineering for Innovation, University of Salento, Via Monteroni, Lecce - Italy
}

\begin{abstract}
Dynamic aeroengine model plays a key role in the design of engine control systems. Moreover, modelling of the engine using performance simulations is an important step in the design process in order to reduce costs, decrease risks and shortening development period. Parameters such as engine spool speeds, vibration, oil temperature, exhaust gas temperature, and fuel flow are often used to estimate performance in gas turbine engines. In this study, two artificial neural network methods were used for the prediction, under transient operations, of one of the most important engine parameters, the Exhaust Gas Temperature (EGT).

The data used for model training are time series datasets of several different flight missions, which have been created using a gas path analysis, and that allow to simulate the engine transient behaviour. The study faces the challenge of setting up a robust and reliable Nonlinear Input-Output (NIO) and a Nonlinear AutoRegressive with eXogenous inputs (NARX) models, by means of a good selection of training.

At the end of the study, two network that predicts the engine EGT in transient operations with the smallest error have been identified.
\end{abstract}

\section{Introduction}

On-board dynamic models of aeroengines can monitor engine performance parameters for fault diagnosis, performance and deterioration control [1,2,3]. However, aeroengine is a multi-variable, nonlinear and very complex thermodynamics system, operating with variable environment. Therefore, it is of great importance to accurately model the behaviour of the engine in its whole flight envelope. However, it is still essential to implement an on-board model embedded in FADEC (Full Authority Digital Electronic Controller) in order to track in real-time the engine performance [1].

In the literature $[5,6,7]$ engine performance simulations have been extensively used in modern gas turbine engine designs in order to decrease design and development costs. These types of simulations can be classified into design point, off-design steady state, and transient performance simulations based on the engine's operation. Engine transient performance simulations are very useful in the initial stage of engine design. Due to the difficulties in the development of a detailed and accurate mathematical model of the aeroengine, data-driven methods are of great interest in the aviation engine community.

*mariagrazia.degiorgi@unisalento.it 
They use real data or experimental data to represent and model the performance of the components and predict the transient behaviour of the system [2].

In the recent past artificial neural networks (ANN) have been widely used as datadriven model for modeling and simulation of aeroengine performance. ANN itself includes different approaches such as Nonlinear AutoRegressive with eXogenous inputs (NARX), feedforward Multi-Layer Perceptron (MLP), BackPropagation Neural Networks (BPNN) and Radial Basis Function (RBF). NARX model, as a recurrent neural network, has the capability of capturing dynamics of complicated systems such as gas turbines [8].

The aim of this paper is to study and predict the transient behaviour of the Viper 632-43 turbojet engine with artificial neural networks, in particular using NIO and NARX neural networks. The engine model network considers engine measurable data without any information about its health status; so, this ANNs considers engine operating condition and gives as output engine measurable data. This first step can be considered an engine model able to monitor the engine performance. Data coming from ANNs are compared with the those from real engine acquisition system, in order to validate networks performance.

\section{Artificial Neural Networks for aeroengine EGT prediction}

ANN rely on real data from the system in order to learn and predict future state of the engine. The interest in application and use of neural networks in health monitoring and prognosis of aircraft gas turbine engine is mainly due to their capability in modelling nonlinearities of dynamical systems. Neural networks provide reasonable tools for nonlinear problems and modelling complex and dynamical systems with great flexibility. Implementation of neural networks in engine fault diagnosis have been extensively presented in the literature. These computational intelligence-based methods have been used for fault diagnostic and prognostic yet [9].

A neural network is a system inspired by the architecture of biological nervous systems. It consists of interconnected neurons (processing units), which are organized in a sequence of layers, including an input layer, one or more intermediate hidden layers, and an output layer. The input signals, with different intensity and strength, enter the neuron and combine to form a net input into another neuron. The output layer, with a number of neurons equal to the number of expected outputs, is calculated by weight and bias associated with connections among neurons. The intermediate layers are connected to the input and output layers. Each neuron in the hidden and output layers receives the signals from all the neurons of the previous layer and then performs a weighted sum on inputs. An example of a feed-forward neural network with one hidden layer is shown in Figure 1.

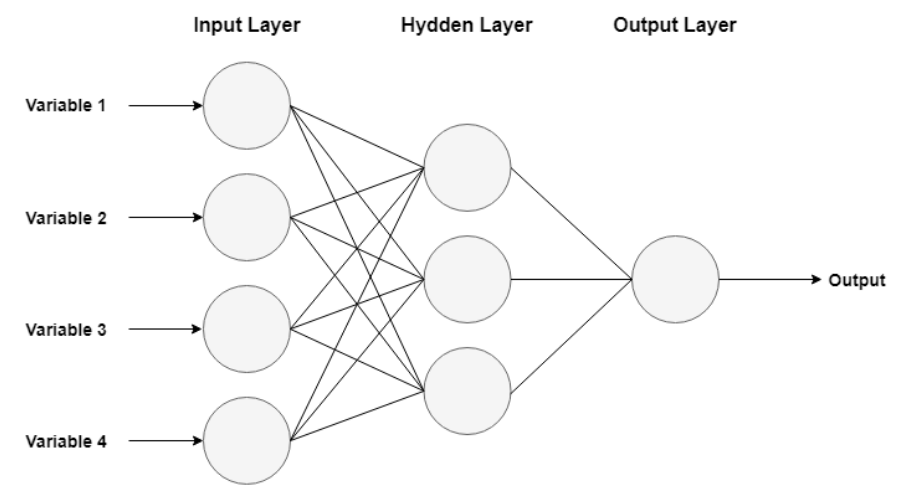

Figure 1. Feed-forward net with one hidden layer (3 neurons) 
Among ANN based models, the most promising approach for the prediction of transient behaviour of jet engine is given by NIO and NARX. These neural networks are suitable for capturing dynamics of complicated systems such as gas turbines, so it can be applied for design optimization of gas turbines, as well as of the whole operation and maintenance activity of the aeroengine.

\subsection{Nonlinear Input-Output neural network}

Nonlinear Input-Output are networks that are useful for system identification and time series prediction. These networks should be trained using a single training dataset, containing a time series indicated with $x(t)$ to underline the time dependence of the data. The structure of the neural network can be assumed as a "black box" with the $x(t)$ series at the input and the $y(t)$ series at the output, which is the variable that ANN should predict. The NIO networks has a delay, indicated with $d$; such neural networks predict the value of the series $y(t)$, at the time $t$, using the $d$ past values of series $x(t)$. The NIO neural network used in this paper is shown in Errore. L'origine riferimento non è stata trovata..

$$
y(t)=f(x(t-1), \ldots, x(t-d))
$$

In this paper the EGT has been selected as the main parameter to be estimated using ANNs; that is, the EGT value is the output $y(t)$. It is necessary to provide as input some parameters that are thought to have a strong influence on the EGT. Between all the available variables, six parameters have been selected, which are considered to have a correlation with EGT.

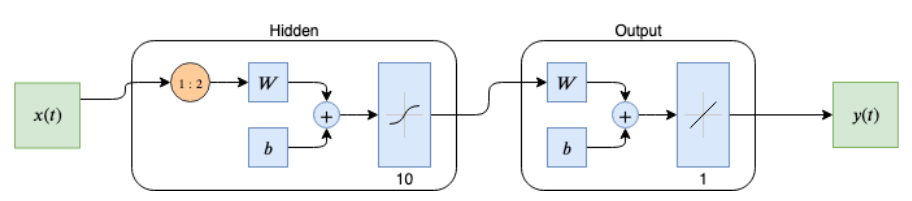

Figure 2 NIO neural network used in this work

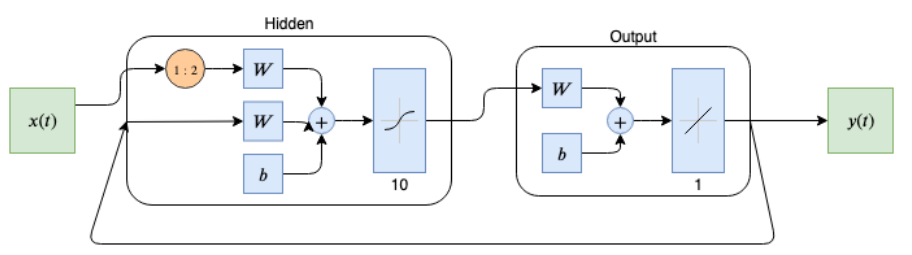

Figure 3 NARX neural network used in this work

In particular, the following ones have been chosen:

- Environment parameters: Mach number, M; Atmospheric total temperature, $\mathrm{T}_{\mathrm{t} 1}$; Atmospheric total pressure, $p_{t 1}$

- Engine parameters: Shaft speed, $N$; Turbine inlet total temperature, $\mathrm{T}_{\mathrm{t} 4}$; Fuel mass flow rate, $\mathrm{W}_{\mathrm{f}}$

Three of these parameters are associated to the flight environment and the others are associated to the engine; this choice was made to study how variables of different types affect the EGT value and especially if it has some correlation with the properties of the air during the flight, which is not obvious.

In order to study how each of these 6 variables influences the EGT, a sensitivity analysis was carried out using 11 combinations of these parameters as input dataset; the process was started by providing, in the first configuration, a single parameter and then 
gradually increasing their number. In general, the increase in the number of inputs has a beneficial effect on the final solution; the greater the number of provided variables, the more the model will be consistent with the data to be predicted.

In order to run the NIO networks, it was decided to use, as training dataset, the parameters from 5 flight missions calculated by transient analysis of a 0 -D model of the Viper engine produced with the software GSP, more details are given in [3]. The networks (one for each configuration) have been trained with Bayesian regularization back propagation training algorithm function; each network has one hidden layer with 10 hidden neurons and a delay of 2 , as in Figure 2Errore. L'origine riferimento non è stata trovata.. After the neural networks have been trained, they were tested, i.e. validated, using as input the same parameters but belonging to 3 new flight missions completely unknown to the network. The prediction carried out is of the "one-step ahead" type, which means that the network predicts the EGT value at the time $t+1$ knowing the values of the inputs $x$ in the previous instants.

\subsection{NARX neural network}

NARX represent an improvement of NIO networks, as they allow to use as input not only the series $x(t)$ series but also the values of the series $y(t)$ in the past times. That is, NARX are recurrent dynamic networks that correlate the current value assumed by an output parameter $y(t)$ in a time series to the past values of the same parameter and of the driving parameters. The NARX neural network used in this paper is shown in Errore. L'origine riferimento non è stata trovata.. The defining equation that relates the next value of the dependent output signal $y(t)$ to the previous values of the output signal $y$ and previous values of the independent signal $x$ is:

$$
y(t)=f(x(t-1), \ldots, x(t-d), y(t-1), \ldots, y(t-d))
$$

The advantage of NARX network with respect to other recurrent network is that it converges easier and needs less training cycles [10]. NARX model provides a description of the system in terms of a nonlinear function of delayed input, output, and prediction error. Hence NARX networks can generalize any nonlinear dynamical system and can be applied in several fields such as prediction, time-series modelling, chaotic time-series prediction, nonlinear filtering, control and modelling etc [11]. NARX solutions are more accurate than other ones such as NIO networks and, thanks to the recurrent nature, they typically require less time for training and much fewer input parameters. The procedure for the training and the validation of NARX is the same as for NIO networks.

\section{Results and discussion}

\subsection{NIO neural network}

Table 1 illustrates the 11 configurations that have been tested, with the relative inputs used. The table also lists the performance (overall, training and testing) of the neural network and the coefficient of determination $R$. It can be noticed that, in the cases in which the inputs are only those relative to flight conditions, both the training phase and the test phase (the prediction) of the network have completely unacceptable performances; as a direct consequence, the value of $R$ is significantly lower than 1 . From configuration $n^{\circ} 5$ onward the parameters associated with the engine operation have been considered. In this case, the NIO performance improves significantly, and $R$ reaches values higher than $97 \%$. Among them, the parameter that most influences the EGT value appears to be the fuel flow rate $\mathrm{W}_{\mathrm{f}}$ whose, used in combination with the shaft rotation speed $N$ guarantees the maximum $R$ value (configuration $n^{\circ}$ ). 
Table 1 Nonlinear Input-Output nets performance during the training on the 5 flight missions

\begin{tabular}{|c|c|c|c|c|c|c|c|c|c|c|}
\hline \multirow{2}{*}{$\mathrm{N}^{\circ}$} & \multicolumn{6}{|c|}{ Inputs } & \multicolumn{4}{|c|}{ Results } \\
\hline & Mach & $\mathrm{T}_{\mathrm{t} 1}$ & $\mathrm{p}_{\mathrm{t} 1}$ & RPM & $\mathrm{T}_{\mathrm{t} 4}$ & $\mathrm{~W}_{\mathrm{f}}$ & Ov. Perf & Tr. Perf & Pred Perf & $R$ \\
\hline 1 & $\checkmark$ & $x$ & $x$ & $x$ & $x$ & $x$ & $7.413 \mathrm{e} 3$ & $7.422 \mathrm{e} 3$ & $7.364 \mathrm{e} 3$ & 0.70080 \\
\hline 2 & $x$ & $\checkmark$ & $x$ & $x$ & $x$ & $x$ & $9.835 \mathrm{e} 3$ & $9.772 \mathrm{e} 3$ & $1.019 \mathrm{e} 4$ & 0.56999 \\
\hline 3 & $\checkmark$ & $\checkmark$ & $x$ & $x$ & $x$ & $x$ & $5.840 \mathrm{e} 3$ & $5.740 \mathrm{e} 3$ & $6.407 \mathrm{e} 3$ & 0.77404 \\
\hline 4 & $\checkmark$ & $\checkmark$ & $\checkmark$ & $x$ & $x$ & $x$ & $5.075 \mathrm{e} 3$ & $4.972 \mathrm{e} 3$ & $5.658 \mathrm{e} 3$ & 0.80726 \\
\hline 5 & $\checkmark$ & $\checkmark$ & $\checkmark$ & $\checkmark$ & $x$ & $x$ & 856 & 764 & 1377 & 0.97016 \\
\hline 6 & $\checkmark$ & $\checkmark$ & $\checkmark$ & $x$ & $\checkmark$ & $x$ & 839 & 714 & 1547 & 0.97077 \\
\hline 7 & $\checkmark$ & $\checkmark$ & $\checkmark$ & $x$ & $x$ & $\checkmark$ & 944 & 733 & 2142 & 0.96711 \\
\hline 8 & $\checkmark$ & $\checkmark$ & $\checkmark$ & $\checkmark$ & $\checkmark$ & $x$ & 814 & 661 & 1686 & 0.97166 \\
\hline 9 & $\checkmark$ & $\checkmark$ & $\checkmark$ & $\checkmark$ & $x$ & $\checkmark$ & 792 & 687 & 1386 & 0.97244 \\
\hline 10 & $\checkmark$ & $\checkmark$ & $\checkmark$ & $x$ & $\checkmark$ & $\checkmark$ & 1102 & 634 & 3754 & 0.96183 \\
\hline 11 & $\checkmark$ & $\checkmark$ & $\checkmark$ & $\checkmark$ & $\checkmark$ & $\checkmark$ & 956 & 622 & 2851 & 0.96679 \\
\hline
\end{tabular}

Ov. Perf $=$ Overall Net Performance, Tr. Perf $=$ Training Performance, Pred Perf $=$ Prediction Performance

Figure 4 and Figure 5 show the comparison between the true EGT (calculated by engine model transient simulations) and the one provided by the neural network for configuration $\mathrm{n}^{\circ} 9$, and the committed percentage error. The two curves are very close to each other, which indicates an accurate prediction. The percentage error appears to have very high but still very short peaks (a few seconds); has been verified that these peaks coincide with situations in which the pilot acts on the throttle in an extremely rapid manner, causing the fuel flow rate, and therefore the EGT, to vary in an abrupt and unpredictable manner. In such situations the neural network cannot accurately predict the variation of the EGT, producing high errors. However, it can be observed that there are areas where the error is extremely low; here the pilot does not significantly modify the throttle and the engine works in almost stationary conditions. The neural network is therefore able to predict EGT with great precision in quasi-steady or weakly unsteady flight conditions.

The average percentage error in the three test mission is equal to $9.22 \%$. However, looking at Figure 5, it can be seen that there are a large number of records that exceed a certain error threshold (in this case $+/-10 \%$ ), which indicates that the networks predict EGT fairly accurately but not without difficulty.

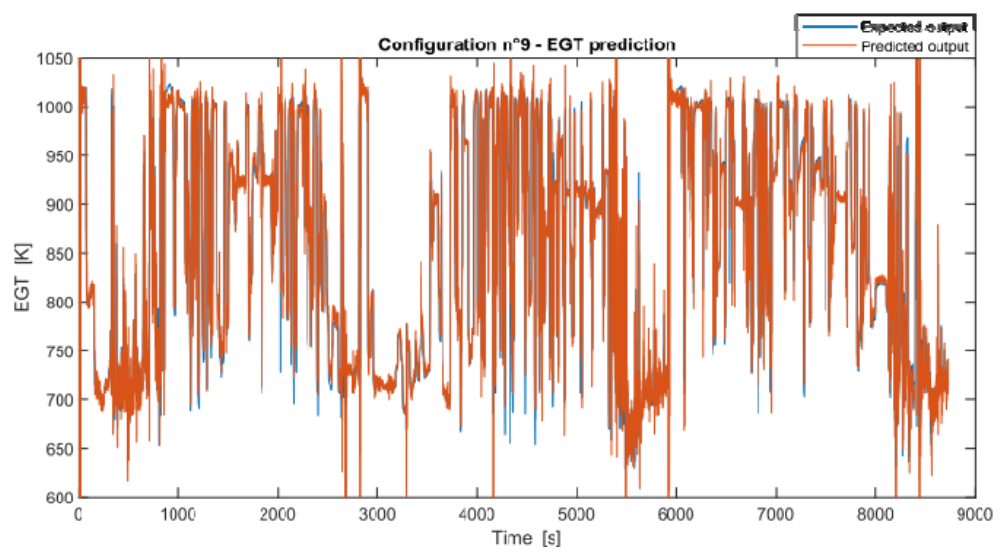


Figure 4 Nonlinear Input-Output network EGT prediction (configuration $n^{\circ} 9$ ) with regards to the test on the three flight missions

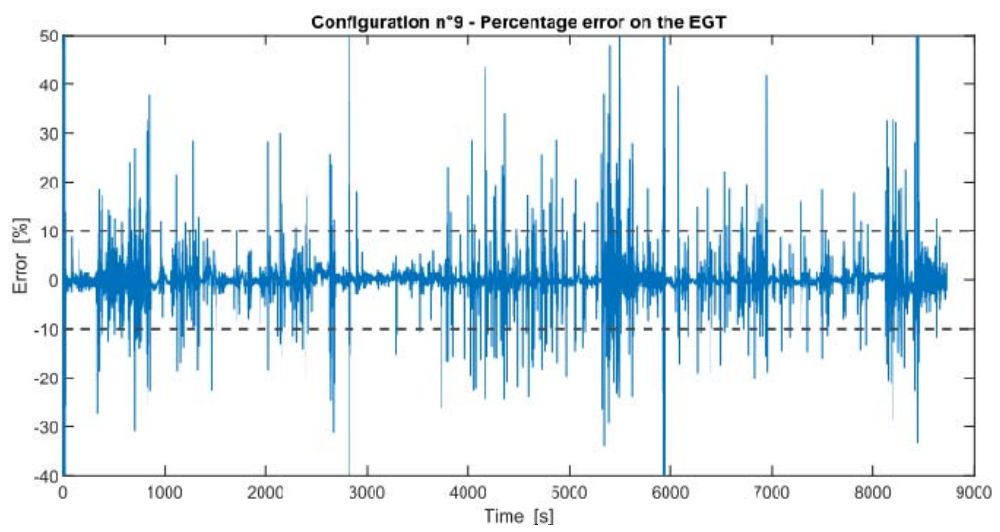

Figure 5 NIO percentage error on the EGT prediction (configuration $n^{\circ} 9$ ) with regards to the test on the three flight missions. Dashed lines indicate an error threshold of $+/-10 \%$

\subsection{NARX neural network}

The same 11 input configurations were used for the NARX networks. Table 2 shows the results, as for the previous validation. In this case, the advantage of using NARX networks is immediately clear: thanks to the recursive use of past EGT values, the training of these networks is very efficient, even if only one parameter not linked to the engine operation is used, such as the Mach number. In fact, using the previous EGT values means that network training always takes place using at least one parameter related to the engine, making it in fact much more effective compared to that of NIO networks.

The predictions made by the network for the 3 testing missions have also proved to be extremely reliable, with a correlation between true and predicted values of about $97 \%$ for all the configurations. In this way it is possible to train a NARX network, and to predict key engine's parameters, using very few inputs for the training.

In the case of NARX networks it is therefore not clearly identifiable the best configuration because the prediction performance is very similar to each other. In order to observe how reliable these are, Figure 6 and Figure 7 show the EGT prediction and the percentage error for the first configuration concerning the three test flight missions.

It is evident the good agreement between the predicted EGT and the real data. In particular, the average percentage error for the EGT prediction is $12.57 \%$; although this value is slightly higher than that for NIO networks, it is worth noting that the number of points with a percentage error of more than $10 \%$ (or less than $-10 \%$ ) is only 38 , compared to 400 of the previous case as can be seen in Figure 7.

Table 2 NARX nets performance during the training on the 5 flight missions

\begin{tabular}{|c|c|c|c|c|c|c|c|c|c|c|}
\hline \multirow{2}{*}{$\mathrm{N}^{\circ}$} & \multicolumn{6}{|c|}{ Inputs } & \multicolumn{4}{|c|}{ Results } \\
\hline & Mach & $\mathrm{T}_{\mathrm{t} 1}$ & $\mathrm{p}_{\mathrm{t} 1}$ & RPM & $\mathrm{T}_{\mathrm{t} 4}$ & $\mathrm{~W}_{\mathrm{f}}$ & Ov. Perf & Tr. Perf & Pred Perf & $\mathrm{R}$ \\
\hline 1 & $\checkmark$ & $x$ & $x$ & $x$ & $x$ & $x$ & 872 & 847 & 1014 & 0.96960 \\
\hline 2 & $x$ & $\checkmark$ & $x$ & $x$ & $x$ & $x$ & 885 & 874 & 1193 & 0.96913 \\
\hline 3 & $\checkmark$ & $\checkmark$ & $x$ & $x$ & $x$ & $x$ & 857 & 824 & 1047 & 0.97012 \\
\hline 4 & $\checkmark$ & $\checkmark$ & $\checkmark$ & $x$ & $x$ & $x$ & 839 & 861 & 714 & 0.97077 \\
\hline 5 & $\checkmark$ & $\checkmark$ & $\checkmark$ & $\checkmark$ & $x$ & $x$ & 847 & 809 & 1062 & 0.97047 \\
\hline
\end{tabular}




\begin{tabular}{|c|c|c|c|c|c|c|c|c|c|c|}
\hline 6 & $\checkmark$ & $\checkmark$ & $\checkmark$ & $\boldsymbol{X}$ & $\checkmark$ & $\boldsymbol{X}$ & 828 & 800 & 988 & 0.97114 \\
\hline 7 & $\checkmark$ & $\checkmark$ & $\checkmark$ & $\boldsymbol{X}$ & $\boldsymbol{X}$ & $\checkmark$ & 814 & 766 & 1086 & 0.97163 \\
\hline 8 & $\checkmark$ & $\checkmark$ & $\checkmark$ & $\checkmark$ & $\checkmark$ & $\boldsymbol{X}$ & 841 & 768 & 1250 & 0.97071 \\
\hline 9 & $\checkmark$ & $\checkmark$ & $\checkmark$ & $\checkmark$ & $\boldsymbol{X}$ & $\checkmark$ & 820 & 819 & 826 & 0.97143 \\
\hline 10 & $\checkmark$ & $\checkmark$ & $\checkmark$ & $\boldsymbol{X}$ & $\checkmark$ & $\checkmark$ & 857 & 811 & 1119 & 0.97012 \\
\hline 11 & $\checkmark$ & $\checkmark$ & $\checkmark$ & $\checkmark$ & $\checkmark$ & $\checkmark$ & 847 & 763 & 1321 & 0.97050 \\
\hline
\end{tabular}

Ov. Perf $=$ Overall Net Performance, Tr. Perf $=$ Training Performance, Pred Perf $=$ Prediction Performance

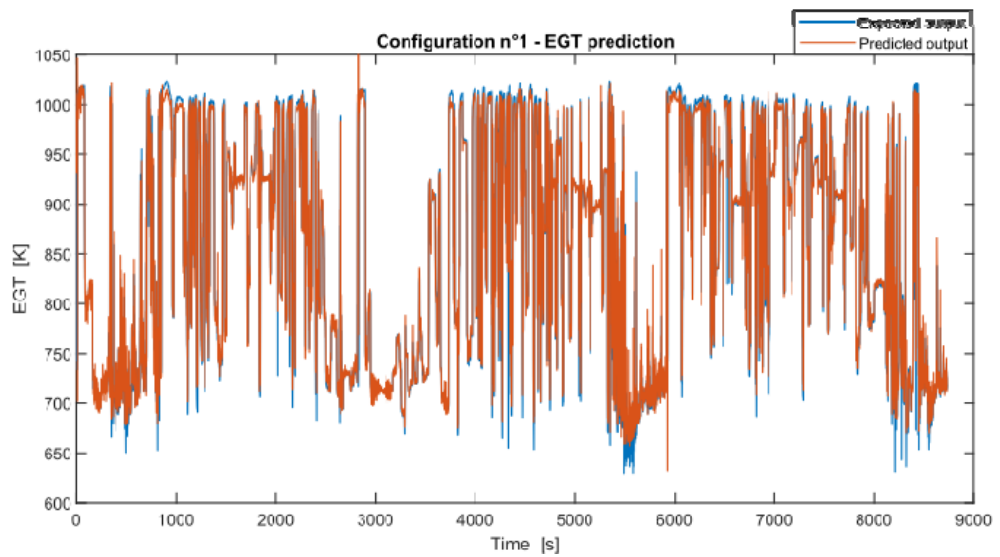

Figure 6 NARX network EGT prediction (configuration $n^{\circ} 1$ ) with regards to the test on the three flight missions

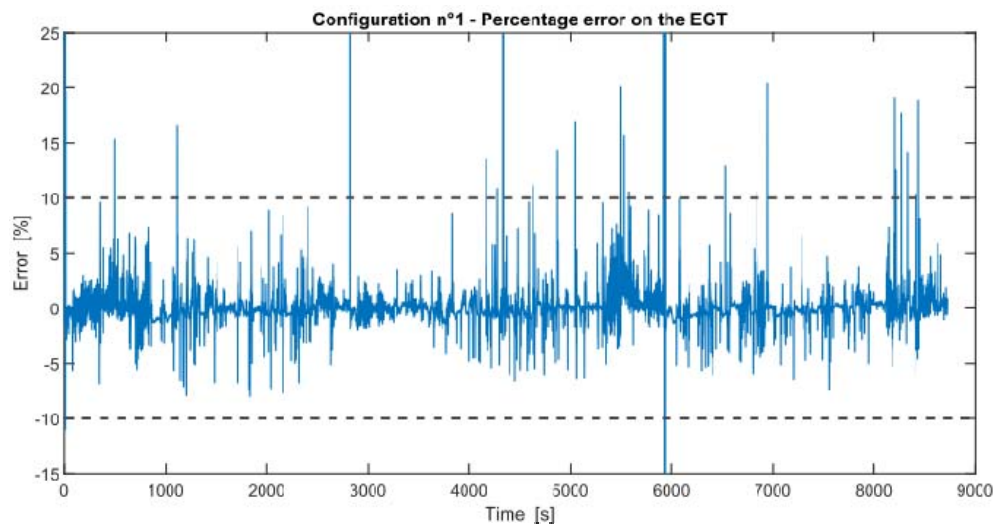

Figure 7 NARX percentage error on the EGT prediction (configuration $n^{\circ} 1$ ) with regards to the test on the three flight missions. Dashed lines indicate an error threshold of $+/-10 \%$

\section{Conclusions}

Concerning NIO networks, it is possible to see how the use of only environment parameters is not sufficient for an accurate EGT forecast; this behaviour is however very intuitive, as it is clearly not possible to successfully predict the exhaust gas temperature without considering such parameters related to the engine operation. The introduction of even a single engine variable increases the forecasting capabilities of NIO networks in an exceptional way, with a correlation between actual and predicted values generally higher than $96 \%$. 
Regarding NARX networks, instead, results show that the predictions made for different flight missions have proved to be extremely reliable, with a correlation between true and predicted values of around $97 \%$ for all the input configurations.

In addition, even the peaks of the prediction percentage error in NARX networks are significantly lower than that in the NIO networks, being reduced from maximum values of more than $50 \%$ to maximum values equal to about $25 \%$. The disadvantage of NARX networks, of course, is that they require the use of output parameters in previous instants of time. However, the output values $y$ may not always be available; in this case one must use NIO networks, anyway with excellent results.

These results suggest that for one-step ahead EGT prediction it may be enough to use only its past values, making it useless to use other inputs. For future work it is therefore suggested to follow this way, using Nonlinear AutoRegressive (NAR) or Long Short-Term Memory (LSTM) networks for the EGT forecast.

\section{References}

[1] Q. Zheng, H. Zhang, Y. Li and Z. Hu, IEEE Access, 6, 45755-45761 (2018)

[2] K. Khorasani, S. Kiakojoori, Neural Comput. Appl., 27, 2157-2192 (2016)

[3] M.G. De Giorgi, A. Ficarella, L. De Carlo, Aircr. Eng. Aerosp. Tec., (to be published)

[4] M.G. De Giorgi, S. Campilongo, A. Ficarella, J. of Eng. for Gas Turbines and Power, 137, 052603-1-052603-15 (2015)

[5] M.G. De Giorgi, S. Campilongo, A. Ficarella, Energy Procedia, 148, 860-867 (2018)

[6] M.G. De Giorgi, S. Campilongo, A. Ficarella, MATEC Web Conf., 233 (2018)

[7] C. Wang, Y.G. Li, B.Y. Yang, Appl. Therm. Eng., 114, 1029-1037 (2017)

[8] H. Asgari, X. Chen, M. Morini, M. Pinelli, R. Sainudiin, P.R. Spina and M. Venturini, Appl. Therm. Eng., 93, 368-376 (2016)

[9] M. Tahan, E. Tsoutsanis, M. Masdi e A. K. Z.A., Appl. Energy, 198, 122-144 (2017)

[10] V. Kodogiannis, P. Lisboa e J. Lucas, Artif. Intell. in Eng., 10, 203-212 (1996)

[11] A. Vatani, Degradation prognostics in gas turbine engines using neural networks, PhD Thesis, Montreal, Québec, Canada (2013) 